\title{
Trace Detection of Metalloporphyrin-Based Coordination Polymer Particles via Modified Surface-Enhanced Raman Scattering Assisted by Surface Metallization
}

\author{
Yu Sun ${ }^{1}$ and Alessio Caravella ${ }^{2}$ \\ ${ }^{1}$ International Institute for Carbon-Neutral Energy Research (WPI-I ${ }^{2}$ CNER), Kyushu University, Motooka 744, Nishi Ku, \\ Fukuoka 819-0395, Japan \\ ${ }^{2}$ Department of Environmental and Chemical Engineering, University of Calabria, Via Pietro Bucci, Cubo 44A, 87036 Rende, Italy \\ Correspondence should be addressed to Yu Sun; ysunyu@i2cner.kyushu-u.ac.jp
}

Received 3 October 2016; Revised 21 November 2016; Accepted 5 December 2016

Academic Editor: Zhangjun Hu

Copyright (C) 2016 Y. Sun and A. Caravella. This is an open access article distributed under the Creative Commons Attribution License, which permits unrestricted use, distribution, and reproduction in any medium, provided the original work is properly cited.

This study proposed a facile method to detect metalloporphyrin-based coordination polymer particles (Z-CPPs) in aqueous solution by modified surface-enhanced Raman scattering (SERS). The SERS-active particles are photodeposited on the surface of Z-CPPs, offering an enhanced Raman signal for the trace detection of Z-CPPs.

\section{Introduction}

Metalloporphyrin are compounds formed by a combination of porphyrin and metal ions, playing important roles in catalysis, fluorescence, sensing, optical imaging, electronics, photochemistry, and biological applications [1-6]. Recently, intensive investigation has been dedicated to morphology controlled synthesis of metalloporphyrin-based coordination polymer particles (CPPs) and several groups reported that diverse metalloporphyrin-based nanoscale/microscale particles can be synthesized through a bottom-up self-assembly process assisted by surfactants, such as Pluronic F127, sodium dodecyl sulfate (SDS), and hexadecyltrimethylammonium bromide (CTAB) [7-14]. Furthermore, it has been reported that metalloporphyrin-containing CPPs can be used for solar hydrogen generation and water purification in the aqueous solutions $[13,14]$. Therefore, it is of significant importance to identify metalloporphyrin-based CPPs in the aqueous solution. In our previous works, a large amount of CPPs powder was required to identify CPPs through the X-ray diffraction (XRD) method, which usually requires complex processes, such as repeated centrifugation and sample drying at low temperature to obtain powder of samples for further identification $[13,14]$.
Metal-organic interactions have been the focus of intense multidisciplinary research in areas such as catalytic chemistry, materials science, and molecular electronics $[15,16]$. Surface-enhanced Raman scattering (SERS) is widely recognized as a powerful tool for studying molecular adsorbates on metal surfaces and provides direct information on metalmolecule interactions, even in IR-opaque media such as aqueous solutions [17]. Typically, SERS is employed for (1) molecule detection in solutions or on substrates due to the molecular adsorption on SERS-active particles or SERSactive substrates $[18,19]$ and (2) molecule aggregates on SERS-active substrates [20]. Nevertheless, identification of self-assembled CPPs in the aqueous solution has been infrequently documented. To the authors' best knowledge, except for our idea proposed recently [21], no Raman data were obtained for CPPs in the solution even though coordination polymers have been intensively investigated. This is probably due to the low concentration of the analyte in the solution. Different from typical SERS, in which SERS-active materials, such as $\mathrm{Au}, \mathrm{Ag}$, and $\mathrm{Cu}$ particles, are usually employed as substrates for molecule adsorption or molecule aggregation, we employ the analyte, here CPPs, as substrate and attached SERS-active materials on their surface to enhance the detection signal in the aqueous solution. 


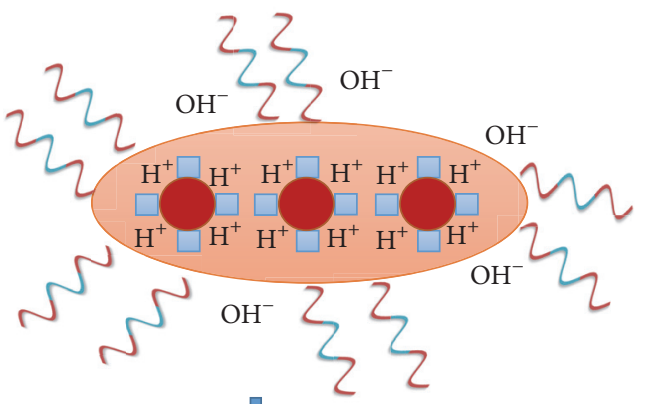

(1) Self-assembly

(2) Injection of $\mathrm{AA}$ and $\mathrm{AuCl}_{4}^{-}$
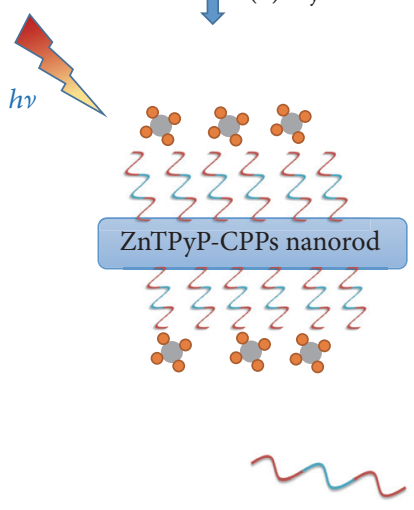

CTAB
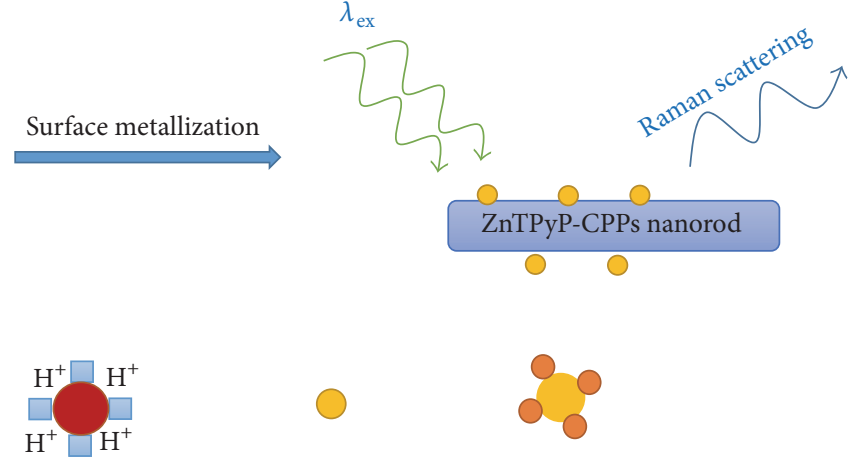

$\mathrm{ZnTPyPH}_{4}{ }^{4+}$

Au nanoparticles

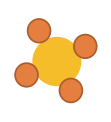

$\mathrm{AuCl}_{4}{ }^{-}$

SCHEME 1: Proposed method for identification of Z-CPPs by SERS through surface metallization in the aqueous solution.

\section{Materials and Methods}

2.1. Materials. Zinc 5, 10, 15, 20-tetra(4-pyridyl)-21H, 23H-porphine (ZnTPyP), cetyltrimethylammonium bromide (CTAB), sodium tetrachloroaurate (III) dehydrate $\left(\mathrm{NaAuCl}_{4} \cdot 2 \mathrm{H}_{2} \mathrm{O}\right)$, ascorbic acid from Aldrich Chemical Co., sodium hydroxide, and hydrochloric acid from Wako Chemicals were used without further purification. All solvents were prepared by using Milli-Q water.

2.2. Preparation of Stock Solutions. As ZnTPyP does not readily dissolve in water, its homogeneous stock solution $(0.01 \mathrm{M})$ was prepared by dissolving an appropriate amount of $\mathrm{ZnTPyP}$ in $\mathrm{HCl}$ solution $(0.2 \mathrm{M})$ to acidify its pyridyl groups, forming soluble tetrapyridium cations. The basic stock solution of surfactants was prepared by dissolving $0.01 \mathrm{M} \mathrm{CTAB}$ and $0.02 \mathrm{M}$ sodium hydroxide in aqueous solution. The gold complex solution $(10 \mathrm{mM})$ was prepared by dissolving $\mathrm{NaAuCl}_{4} \cdot 2 \mathrm{H}_{2} \mathrm{O}$ in $10 \mathrm{~mL}$ aqueous solution. The electron donor solution $(0.1 \mathrm{M})$ was freshly prepared by dissolving ascorbic acid in the aqueous solution.

2.3. Preparation of ZnTPyP-CPPs (Abbreviated as Z-CPPs). An amount of $250 \mu \mathrm{L}$ of a ZnTPyP stock solution (0.01M) was rapidly injected into $5 \mathrm{~mL}$ of a basic stock solution with vigorous stirring at room temperature.

2.4. Preparation of $A u$ Decorated ZnTPyP-CPPs (Abbreviated as $A u-Z-C P P s)$. The solution of gold complexes
$\left(\mathrm{NaAuCl}_{4} \cdot 2 \mathrm{H}_{2} \mathrm{O}\right)$ together with freshly prepared ascorbic acid solution was added to the freshly prepared Z-CPPs solution. After mild stirring for $40 \mathrm{~min}$ under visible light illumination, the color of the mixture solution turned into light-reddish, indicating the successful photoreduction from gold complexes to gold nanoparticles. Raman spectra were recorded without further treatment (Figure 2).

2.5. Characterization. To prepare the samples for fieldemission scanning electron microscopy (FE-SEM, TESCAN, and MIRA3) and transmittance electron microscopy, the asprepared $\mathrm{ZnTPyP}$ particles were redispersed in pure water, dropped onto silicon wafer substrate and $\mathrm{Cu}$ grid, respectively, and finally dried at $50^{\circ} \mathrm{C}$ in the oven. The chemical state of gold element was determined with X-ray photoelectron spectroscopy (XPS, ESCA2000), which was performed with an $\mathrm{Al} \mathrm{K} \alpha$ and $\mathrm{Mg} \mathrm{K} \alpha \mathrm{X}$-ray source. Raman measurements were performed using a Renishaw 2000 Raman microscope system (Renishaw, UK). A Melles Griot HeNe Laser, operating at $\lambda=785 \mathrm{~nm}$, was used as excitation source, with a laser power of approximately $15 \mathrm{~mW}$. The Rayleigh line was removed from the collected Raman scattering using a holographic notch filter located in the collection path. The Raman scattering was collected using a charge-coupled device (CCD) camera at a spectral resolution of $4 \mathrm{~cm}^{-1}$. All spectra were calibrated to the $520 \mathrm{~cm}^{-1}$ silicon line. A 20x objective lens was used to focus a laser spot on the glass tube. 


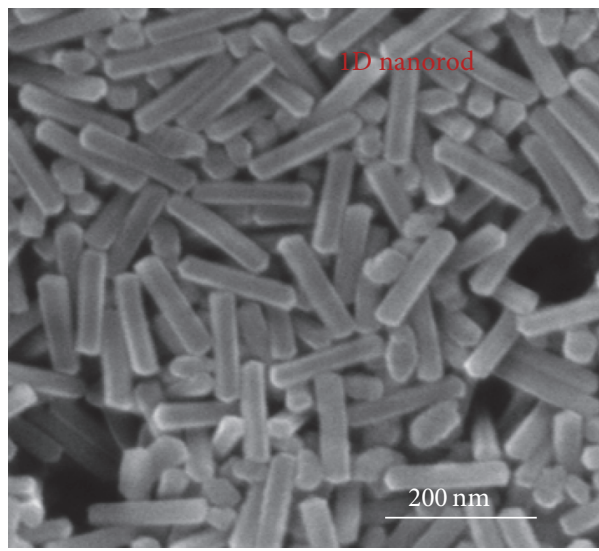

(A)

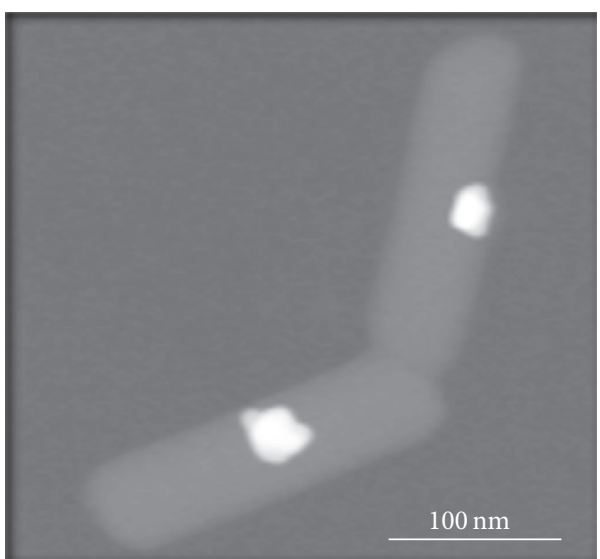

(C)

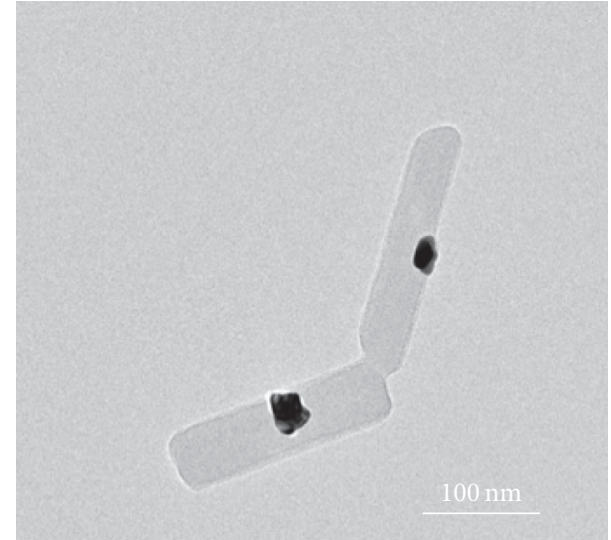

(B)

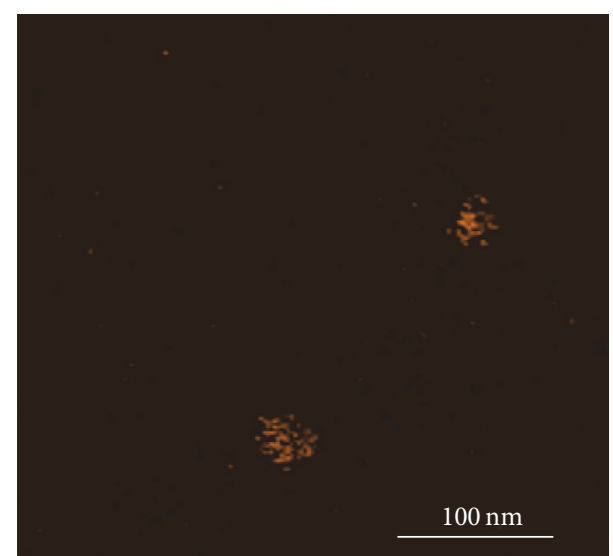

(D)

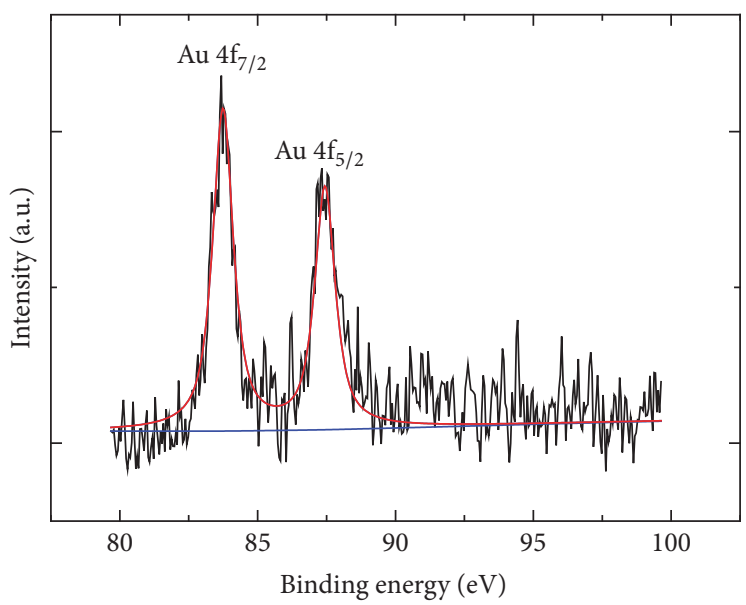

(E)

FIGURE 1: (A) SEM image of the Z-CPPs nanorod structures; TEM (B) and STEM (C) images of Au-Z-CPPs; elemental mapping data of (C) $\mathrm{Au}$ on ZnTPyP-CPPs; (E) XPS analysis of Au element for Au-Z-CPPs.

\section{Results and Discussion}

Briefly, zinc 5, 10, 15, 20-tetra(4-pyridyl)-21H, $23 H$-porphine (abbreviated to ZnTPyP) was chosen for the investigation and $250 \mu \mathrm{L}$ of a ZnTPyP stock solution $(0.01 \mathrm{M})$ was rapidly injected into $5 \mathrm{~mL}$ of a basic stock solution at room temperature and under vigorous stirring. The solution turned cloudy immediately. However, in order to facilitate the growth of ZnTPyP-CPPs (abbreviated to Z-CPPs), the cloudy solution was kept at room temperature without stirring for $12 \mathrm{~h}$. 


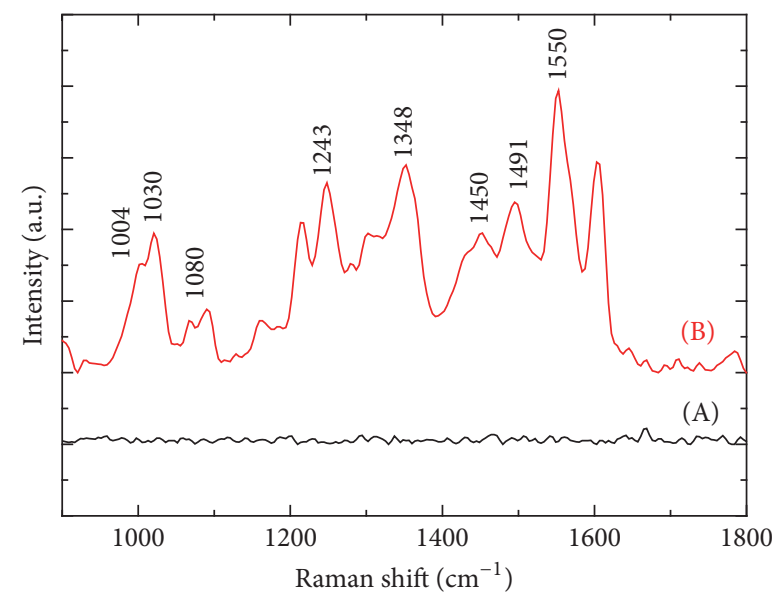

Figure 2: Raman spectra of (A) Z-CPPs and (B) Au-Z-CPPs in the aqueous solution.

Then, we first measured the Raman spectrum of the assynthesized Z-CPPs in the solution without any further treatment; however, no Raman signal was obtained.

To design a method to detect Z-CPPs in the solution, we hypothesized that if SERS-active particles, such as Au particles, can attach on the surface of Z-CPPs, the trace detection could be possibly achieved in the aqueous solution. It was documented that the absorption of visible or UVlight by photocatalysts (PCs) yields the long-lived excited triplet $\pi-\pi$ state, $\mathrm{PCs}^{*}$, which is rapidly reduced by an electron donor (ED) such as ascorbic acid; if the long-lived radical anion has suitable electronic structures, it is capable of efficiently reducing a variety of metal ions including $\mathrm{Ag}, \mathrm{Au}$, $\mathrm{Hg}, \mathrm{Pb}, \mathrm{Cu}$, and $\mathrm{Pt}$ to the zero-valent metals [10]. Therefore, as shown in Scheme 1, immediately after the synthesis of ZCPPs, $0.5 \mathrm{~mL}$ of $\mathrm{NaAuCl}_{4} \cdot 2 \mathrm{H}_{2} \mathrm{O}(10 \mathrm{mM})$ aqueous solution and $1 \mathrm{~mL}$ of ascorbic acid $(0.1 \mathrm{M})$ aqueous solution were add to the Z-CPPs solution. The resulting mixture was gently stirred for $40 \mathrm{~min}$ under visible light illumination until the mixture turned into a light-reddish color.

The external morphologies of the synthesized Z-CPPs and Z-CPPs attached with gold particles (Au-Z-CPPs) were characterized by scanning electron microscopy (SEM) and transmittance electron microscopy (TEM). As shown in Figure 1(A), Z-CPPs nanorods with an average length of $\sim 230 \mathrm{~nm}$ and width of $\sim 80 \mathrm{~nm}$ were synthesized. The TEM image and STEM image (Figures $1(\mathrm{~B})$ and $1(\mathrm{C})$ ) of Au-Z-CPPs together with Au elemental mapping (Figure 1(D)) revealed that $\mathrm{AuCl}_{4}{ }^{-}$complexes were reduced to $\mathrm{Au}$ nanoparticles under visible light illumination, which successfully attached on the surface of Z-CPPs. XPS analysis of Au element for collected powder samples (powder Au-Z-CPPs) is shown in Figure 1(E), which further confirmed that $\mathrm{AuCl}_{4}{ }^{-}$were fully reduced to Au nanoparticles by observing XPS peaks at $83.8 \mathrm{eV}$ and $87.3 \mathrm{eV}$, corresponding to $\mathrm{Au} 4 \mathrm{f}_{7 / 2}$ and $\mathrm{Au} 4 \mathrm{f}_{5 / 2}$ of metallic gold, respectively. The $1 \mathrm{D}$ Z-CPPs maintained their original structure after Au decoration.

As mentioned above, no Raman signal was obtained for $\mathrm{Z}$-CPPs in the aqueous solution (Figure 3(A)). After Au decoration on the surface of the Z-CPPs nanorods, we carried

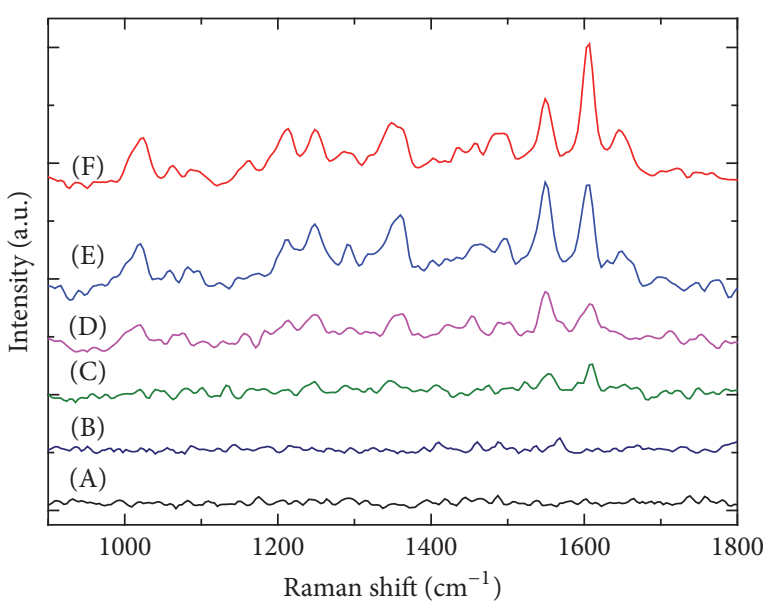

FIgURE 3: SERS spectra of Z-CPPs and Au-Z-CPPs according to the ZnTPyP molecule concentration varying from 500 to $10 \mathrm{nmol}$ : (A) original Z-CPPs solution, Au-Z-CPPs with (B) $10 \mathrm{nmol}$, (C) $20 \mathrm{nmol}$, (D) $100 \mathrm{nmol}$, (E) $200 \mathrm{nmol}$, and (F) $500 \mathrm{nmol}$ of ZnTPyP molecules.

out Raman spectroscopy measurement. It is illustrated that all characteristic group contributions (fingerprints) consistent with $\mathrm{ZnTPyP}$ structures were identified, including $1004 \mathrm{~cm}^{-1}$ $\left[\nu\left(\mathrm{C}_{\alpha}-\mathrm{C}_{\mathrm{m}}\right)\right], 1030 \mathrm{~cm}^{-1}$ [pyrr $\left.\delta(\mathrm{C}-\mathrm{H})\right], 1080 \mathrm{~cm}^{-1}\left[\delta\left(\mathrm{C}_{\beta^{-}}\right.\right.$ $\mathrm{H})], 1243 \mathrm{~cm}^{-1}\left[\nu\left(\mathrm{C}_{\mathrm{m}}-\mathrm{Pyrr}\right)\right]$ as well as the $\varphi$ stretch normal modes, $1384 \mathrm{~cm}^{-1}\left[\nu\left(\mathrm{C}_{\alpha}-\mathrm{H}\right)\right], 1450 \mathrm{~cm}^{-1}$ and $1491 \mathrm{~cm}^{-1}$ $\left[\nu\left(\mathrm{C}_{\alpha}-\mathrm{C}_{\beta}\right)\right]$, and $1550 \mathrm{~cm}^{-1}\left[\nu\left(\mathrm{C}_{\alpha}-\mathrm{C}_{\beta}\right)\right.$ and $\left(\mathrm{C}_{\beta}-\mathrm{C}_{\beta}\right)$ stretch $]$ [20]. This proved that our proposed method, SERS assisted by surface metallization on Z-CPPs (SM-SERS), is an effective approach for the detection of Z-CPPs in the aqueous solution.

In previous reports, we investigated morphology controlled synthesis of Z-CPPs and confirmed that the morphological transformation is related to solute concentration, temperature, and $\mathrm{pH}[13,14]$. Therefore, the investigation of the limit of detection for Z-CPPs through our SM-SERS method was carried out by diluting the $\mathrm{Au}-\mathrm{Z}$-CPPs aqueous solution in order to maintain its original structure. It is worth mentioning that dilution of the Au-Z-CPPs solution also causes a decrease in the concentration of active Au particles. Nevertheless, Figure 3 shows the SERS spectra of the Au-ZCPPs aqueous solution corresponding to ZnTPyP molecule concentrations ranging from 500 to $10 \mathrm{nmol}$. As a reference, the SERS spectrum of the Z-CPPs aqueous solution is also shown in Figure 3(A). It was observed that the signal intensity decreased as the Z-CPPs concentration decreased and the limit detection was $10 \mathrm{nmol}$. This suggested that our SM-SERS method provides a high sensitivity for metalloporphyrinCPPs detection. However, in determining the limit of detection, it was found that no SERS signal could be detected when the Z-CPPs concentration was lower than $10 \mathrm{nmol}$, which is probably due to the decreased concentration of active $\mathrm{Au}$ nanoparticles on the surface of the Z-CPPs.

\section{Conclusion}

In conclusion, we proposed the first demonstration to detect metalloporphyrin-containing CPPs in the aqueous solution 
through a designed SERS method (SM-SERS). Thanks to the photoactive properties of metalloporphyrins, we attached SERS-active gold nanoparticles on the surface of Z-CPPs via photoreduction of gold complexes under visible light illumination. Our proposed method was proven to be effective for Z-CPPs identification in that all characteristic group contributions (fingerprints) were consistent with ZnTPyP structures. The trace detection was achieved by obtaining the Raman spectra with a limit of detection for ZnTPyP of $10 \mathrm{nmol}$.

\section{Competing Interests}

The authors declare that they have no competing interests.

\section{Acknowledgments}

Alessio Caravella gratefully acknowledges the "Programma Per Giovani Ricercatori 'Rita Levi Montalcini”' granted by the "Ministero dell'Istruzione, dell'Università e della Ricerca, MIUR.”

\section{References}

[1] K. Kadish, K. M. Smith, and R. Guilard, Porphyrin Handbook, Academic Press, New York, NY, USA, 1999.

[2] W. M. Campbell, K. W. Jolley, P. Wagner et al., "Highly efficient porphyrin sensitizers for dye-sensitized solar cells," Journal of Physical Chemistry C, vol. 111, no. 32, pp. 11760-11762, 2007.

[3] C. Yao, L.-K. Yan, W. Guan, C.-G. Liu, P. Song, and Z.-M. Su, "Prediction of second-order optical nonlinearity of porphyrinmetal- polyoxometalate sandwich compounds," Dalton Transactions, vol. 39, no. 33, pp. 7645-7649, 2010.

[4] S. Thyagarajan, T. Leiding, S. P. Årsköld, A. V. Cheprakov, and S. A. Vinogradov, "Highly non-planar dendritic porphyrin for pH sensing: observation of porphyrin monocation," Inorganic Chemistry, vol. 49, no. 21, pp. 9909-9920, 2010.

[5] S. M. Riberio, A. C. Serra, and A. M. A. Rocha Gonsalves, "Covalently immobilized porphyrins on silica modified structures as photooxidation catalysts," Journal of Molecular Catalysis A: Chemical, vol. 326, no. 1-2, pp. 121-127, 2010.

[6] A. Verma, S. L. Facchina, D. J. Hirsch et al., "Photodynamic tumor therapy: mitochondrial benzodiazepine receptors as a therapeutic target," Molecular Medicine, vol. 4, no. 1, pp. 40-45, 1998.

[7] V. E. Yushmanov, T. T. Tominaga, I. E. Borissevitch, H. Imasato, and M. Tabak, "Binding of manganese and iron tetraphenylporphine sulfonates to albumin is relevant to their contrast properties," Magnetic Resonance Imaging, vol. 14, no. 3, pp. 255261, 1996.

[8] K. Komagoe and T. Katsu, "Porphyrin-induced photogeneration of hydrogen peroxide determined using the luminol chemiluminescence method in aqueous solution: a structure-activity relationship study related to the aggregation of porphyrin," Analytical Sciences, vol. 22, no. 2, pp. 255-258, 2006.

[9] F. Bai, H. Wu, R. E. Haddad et al., "Monodisperse porous nanodiscs with fluorescent and crystalline wall structure," Chemical Communications, vol. 46, no. 27, pp. 4941-4943, 2010.
[10] F. Bai, Z. C. Sun, H. M. Wu et al., "Porous one-dimensional nanostructures through confined cooperative self-assembly," Nano Letters, vol. 11, no. 12, pp. 5196-5200, 2011.

[11] J.-S. Hu, Y.-G. Guo, H.-P. Liang, L.-J. Wan, and L. Jiang, "Three-dimensional self-organization of supramolecular selfassembled porphyrin hollow hexagonal nanoprisms," Journal of the American Chemical Society, vol. 127, no. 48, pp.17090-17095, 2005.

[12] W. Sun, H. Wang, D. Qi et al., “5,10,15,20-tetra(4-pyridyl)porphyrinato zinc coordination polymeric particles with different shapes and luminescent properties," CrystEngComm, vol. 14, no. 22, pp. 7780-7786, 2012.

[13] Y. Sun and B. Yoo, "Homogenous four-petal flower structure formation from metalloporphyrin self-assembly and its reversible transformation into an octahedron structure," CrystEngComm, vol. 16, no. 38, pp. 8950-8953, 2014.

[14] Y. Sun and B. Y. Yoo, "Morphological transformation reactions of photocatalytic metalloporphyrin-containing coordination polymer particles from seed structures," ChemistryOpen, vol. 4, no. 4, pp. 438-442, 2015.

[15] A. Ulman, "Formation and structure of self-assembled monolayers," Chemical Reviews, vol. 96, no. 4, pp. 1533-1554, 1996.

[16] D. J. Wold and C. D. Frisbie, "Fabrication and characterization of metal-molecule-metal junctions by conducting probe atomic force microscopy," Journal of the American Chemical Society, vol. 123, no. 23, pp. 5549-5556, 2001.

[17] J. Hu, M. Tanabe, J. Sato, K. Uosaki, and K. Ikeda, "Effects of atomic geometry and electronic structure of platinum surfaces on molecular adsorbates studied by gap-mode SERS," Journal of the American Chemical Society, vol. 136, no. 29, pp. 10299-10307, 2014.

[18] K. Ikeda, J. Sato, and K. Uosaki, "Surface-enhanced Raman scattering at well-defined single crystalline faces of platinum-group metals induced by gap-mode plasmon excitation," Journal of Photochemistry and Photobiology A: Chemistry, vol. 221, no. 2-3, pp. 175-180, 2011.

[19] K. Ikeda, J. Sato, N. Fujimoto, N. Hayazawa, S. Kawata, and K. Uosaki, "Plasmonic enhancement of raman scattering on nonSERS-active platinum substrates," Journal of Physical Chemistry C, vol. 113, no. 27, pp. 11816-11821, 2009.

[20] R. Cristescu, C. Popescu, A. C. Popescu et al., "Functional porphyrin thin films deposited by matrix assisted pulsed laser evaporation," Materials Science and Engineering: B, vol. 169, no. 1-3, pp. 106-110, 2010.

[21] Y. Sun and A. Caravella, "Identification of metalloporphyrincontaining coordination polymer particles by surface-enhanced raman scattering through surface metallization," in Proceedings of the 7th International Conference on Nanomaterials-Research and Application (Nanocon '15), Brno, Czech Republic, October 2015. 

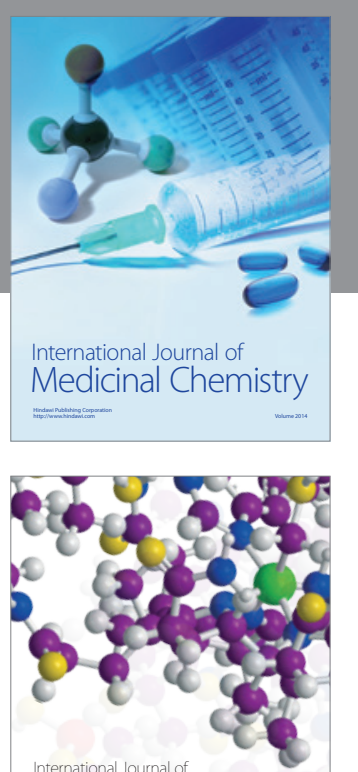

Carbohydrate Chemistry

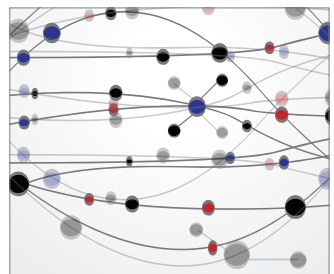

The Scientific World Journal
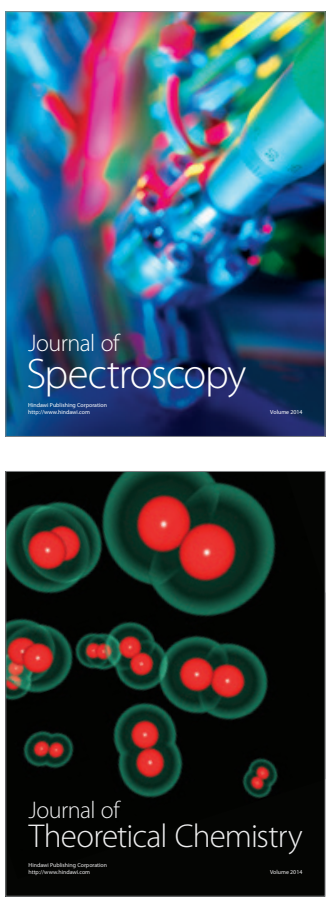
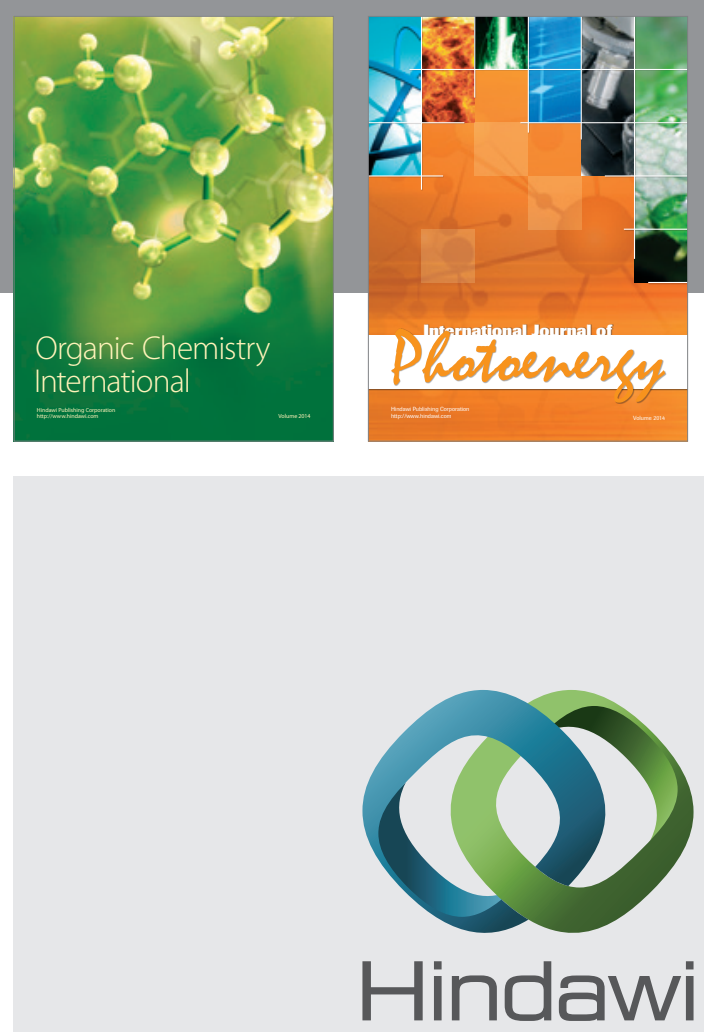

Submit your manuscripts at

http://www.hindawi.com

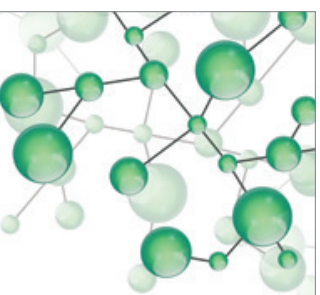

International Journal of

Inorganic Chemistry

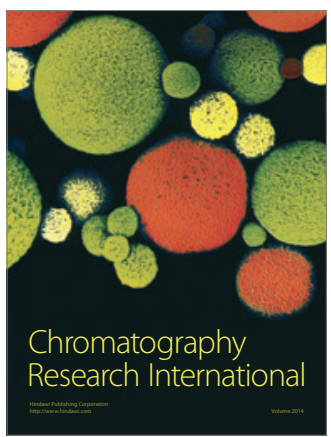

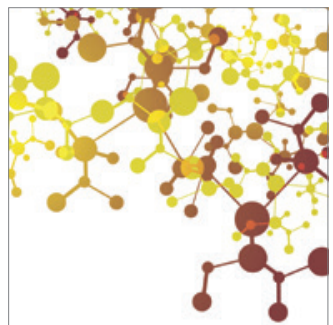

Applied Chemistry
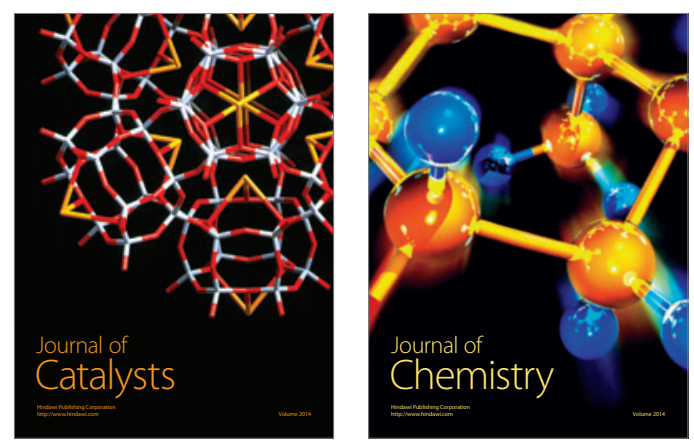
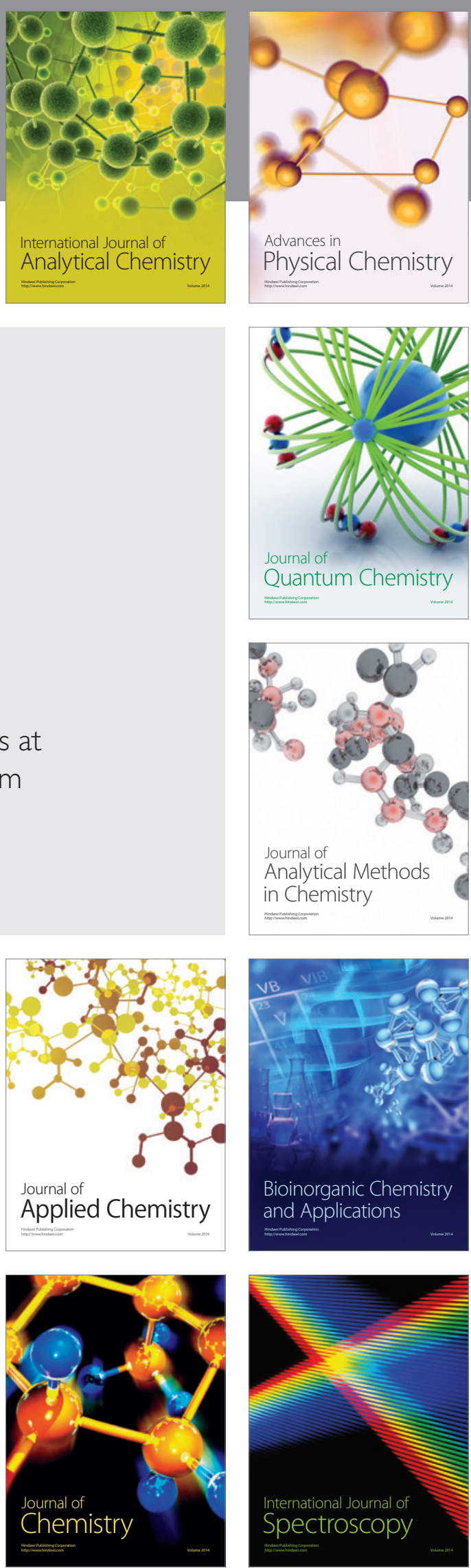